\title{
THE BRONCHOGRAPHIC APPEARANCE OF "PERIPHERAL POOLING" ATTRIBUTED TO THE FILLING OF CENTRILOBULAR EMPHYSEMATOUS SPACES
}

\author{
BY \\ J. G. LEOPOLD AND R. M. SEAL \\ From the Pathology Departments of the Welsh National School of Medicine and Sully Hospital, \\ Glamorgan
}

(RECEIVED FOR PUBLICATION SEPTEMBER 28, 1960)

The bronchographic appearance of clusters of spherical opacities seen at the periphery of the bronchial tree has been termed "peripheral pooling " by Simon and Galbraith (1953). It was a common abnormality in the bronchograms of the advanced cases of chronic bronchitis they studied, being found in 14 of the 21 bronchograms from patients whose standard radiographs suggested emphysema. No precise explanation of the underlying pathology was attempted.

We have suspected, since first we saw this description of "peripheral pooling" and the illustration which accompanied it, that the localization of the opaque medium might be within emphysematous spaces of the centrilobular variety (Leopold and Gough, 1957). Proof of this has recently been obtained in a case of bronchogenic carcinoma showing well-marked "peripheral pooling" in the bronchograms. Granulomas were caused by the aqueous "dionosil" used, thereby preserving, until the time of lobectomy 15 days later, evidence of the common identity of the "pooled" areas and centrilobular emphysematous spaces.

This seems to be the first recorded case of granulomas due to " dionosil aqueous," and in our view it is significant that this undesirable result has occurred in a lung already damaged by emphysema.

\section{Clinical Presentation}

The subject was a male architect aged 50 years. He had suffered from shortness of breath for at least nine months, increasing in the last three months. He had some anorexia. Partly because of recent contact with a case of pulmonary tuberculosis, he was seen at a chest clinic before being referred to Sully Hospital on November 3, 1959.

He said he had a "smoker's cough" productive of a little mucoid sputum and was in the habit of smoking 30 cigarettes a day. He was also a heavy drinker, regularly consuming 7-8 pints of beer a night.

In the two to three weeks before admission to hospital he was hoarse and had a little trans-sternal pain.

He was of spare build with definite clubbing of the fingers and toes. He had a non-productive cough at this time, and bilateral direct inguinal herniae. Chest expansion was equal on both sides. The breath sounds were impaired over the posterior aspect of the right chest and over the left chest anteriorly close to the sternum. An expiratory wheeze was audible over the left side of the chest. His pulse was regular and the blood pressure measured $140 / 85 \mathrm{~mm}$. $\mathrm{Hg}$. The liver edge was just palpable.

His indirect maximum breathing capacity on the day following admission was 39 litres/min.; the haemoglobin was $83 \%$ and there were 16,000 white cells/c.mm.

Plain Radiographs of Chest.-These showed horizontal ribs, low and flattened diaphragms, and a pendulous heart. There was a $8 \times 5 \mathrm{~cm}$. homogenous, oval opacity in the left upper lung fields and the remainder of the lung fields were translucent with a diminished vascular pattern.

BRONCHOSCOPY AND BRONCHOGRAPHY.-Bronchoscopy was carried out on November 5. The vocal cords, trachea, and carina were found to be normal. Secretions were taken for cytological examination but did not show carcinoma cells.

“Dionosil aqueous," 10-15 ml., was introduced by catheter into the left bronchus for bronchography, as described by Smith, Davies, Rothwell, Lodwig, and Mahoney (1960). The immediate film showed good bronchial filling confined to the left lung and most complete in the upper lobe. There was no obvious bronchial block except possibly in a subdivision of the left apico-posterior bronchus. The posterior division of the left upper lobe was updrawn. "Alveolar" filling did not occur, but the appearance of "peripheral pooling" was seen at the terminations of all the outlined twigs of the upper part of the 
bronchial tree and some of the lower (Fig. 1a and b). A radiograph on November 11 showed that although the contrast medium had completely cleared from the bronchi it remained in the "pooled" areas, where often it now took on a stellate pattern, due to the medium lying in crescents instead of circular masses (Fig. 2). This picture remained little changed until thoracotomy on November 20.

Tновлсотому.-A left postero-lateral thoracotomy was carried out through the fourth interspace. Dense adhesions were found between the left upper lobe and the upper mediastinum, and in the upper lobe was a bulky tumour $13 \mathrm{~cm}$. or so in size, placed centrally, but extending chiefly into the posterior segment. Small, emphysematous bullae were noted in the apical and anterior segment.

Left upper lobectomy was performed and numerous small lymph nodes related to the left upper lobe bronchus were removed with the specimen. The bronchial stump was closed with nylon sutures and covered by a flap of the parietal pleura.

Post-operative Course.-Radiographs taken postoperatively showed poor expansion of the remaining lobe and occasional flecks of the opaque medium were still detectable in the mid-zone of the left chest.

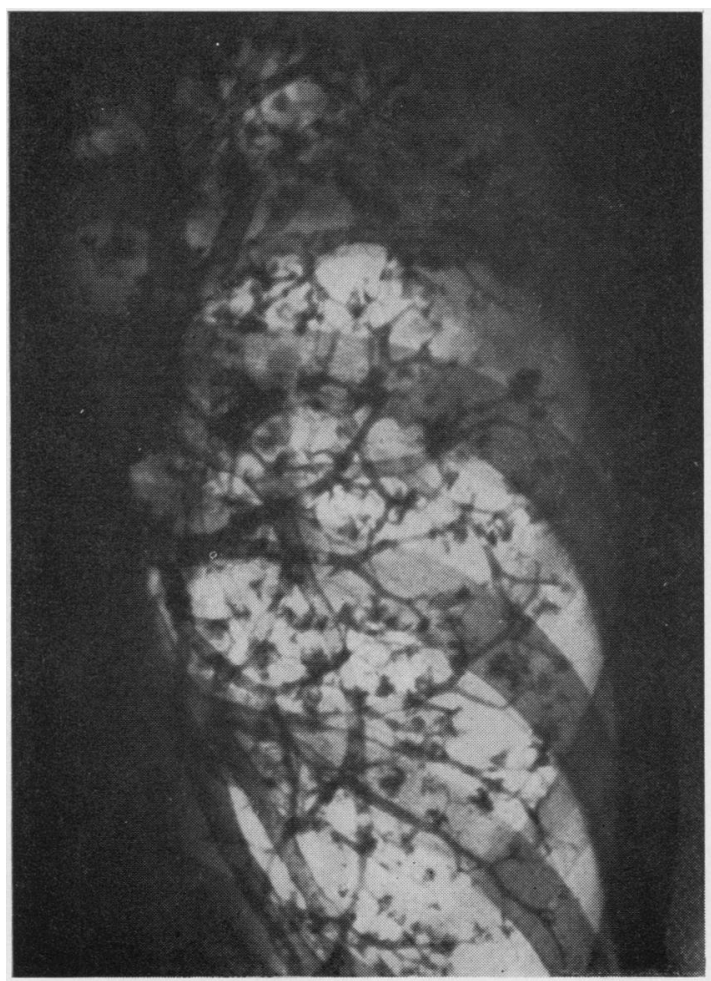

FIG. $1(a)$
The patient's condition gradually declined, respiratory embarrasment became more pronounced, and he died seven days after the operation.

\section{Pathological Findings}

The pathological findings in the resected left upper lobe will be described first, followed by an account of the necropsy findings.

RESECTED LEFT UPPER LOBE. - The specimen showed a large oval, relatively well-circumscribed tumour, $8 \times 6 \times 6 \mathrm{~cm}$., in the apico-posterior segment. Although arising in the territory of the posterior division of the apical bronchus an origin from its surface could not be demonstrated.

Histologically the tumour was a relatively welldifferentiated adenocarcinoma. There was no secretion of mucin. The small nodes at the root of the specimen did not contain tumour.

The remainder of the lobe showed patchy emphysema and scattered throughout were many irregular, firm, grey-coloured nodules from 3 to $4 \mathrm{~mm}$. in diameter. Many of the nodules were seen to be closely related to centrilobular emphysematous spaces. When bisected, the nodules showed a chalky white spot at their centre. Only with frozen sections could

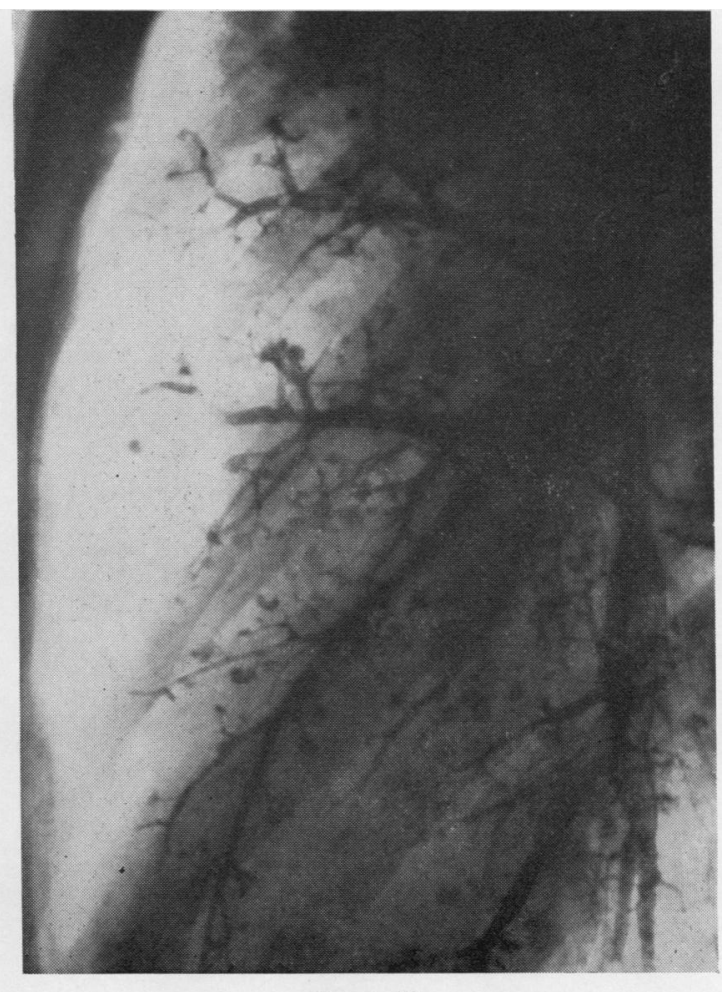

FIG. 1 (b) Fig. 1.-(a) Immediate bronchogram, antero-posterior view. Left mid-zone showing good filling and widespread " peripheral pooling."
O is-third natural size. (b) Immediate bronchogram, lateral view. Anterior segment and lingula showing "peripheral pooling." Onethird natural size. 


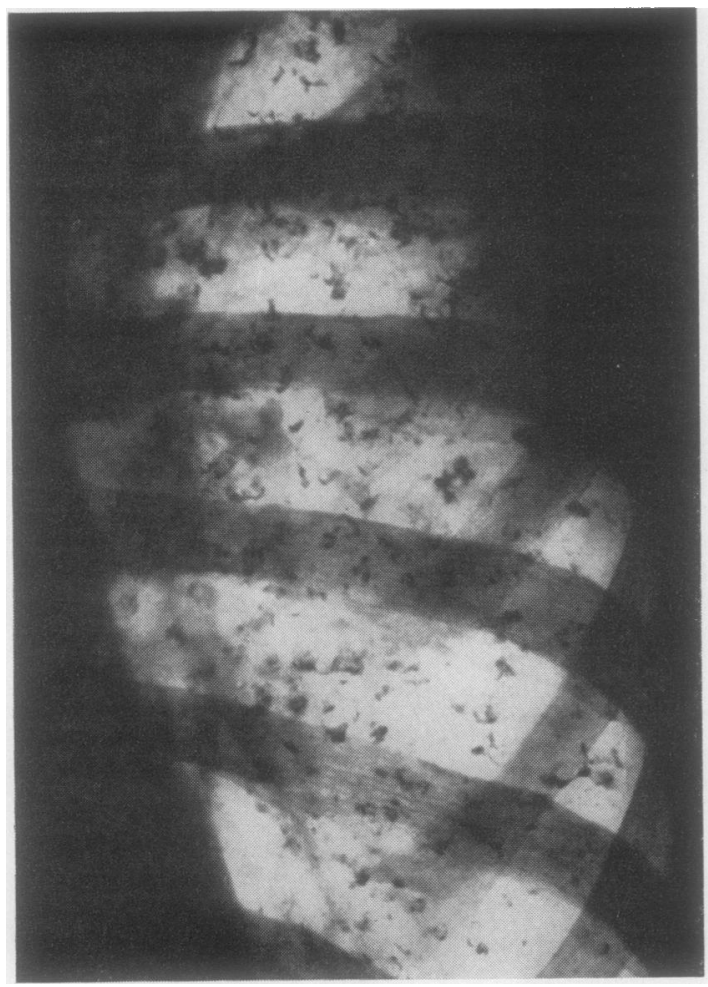

FIG. 2.-Bronchogram six days later, same area as Fig. 1(a). Bronchial outline has cleared isolating the "pooled" areas. The medium now often lies in crescents. Compare upper field with $1(a)$. One-third natural size.

this white material be identified as "dionosil." It appeared as a collection of crystals of greatly varying size up to $30 \mu$ (Fig. 4). Some of the crystals formed rhomboidal plates of nearly equal breadth to length, but the majority had distinct major and minor axes. The crystals found in the granulomas were in every respect similar to the crystals seen in the bronchographic medium used, "dionosil aqueous" (Glaxo) (Fig. 5). The crystals were doubly refractile, and, because of their solubility in xylol, chloroform, and benzene, only the spaces due to them were seen in sections of paraffin-embedded tissue.

Around the central nidus of crystals there was an acellular zone which also showed the outlines of many crystals but had no doubly-refractile content. Carboxymethyl cellulose is another constituent of "dionosil aqueous" which one might expect to be present in the granuloma. Possibly representative of this material were the large irregularly shaped masses of a homogenous substance staining diffusely a faint blue or slate colour with haematoxylin. It was present throughout the acellular area and particularly noticeable at its boundary. At the latter site the material was surrounded by macrophages in syncytial arrangement (Fig. 6). Doubly-refractile material was not found in frozen sections of these giant cells although, once again, crystal spaces occasionally remained visible. The homogenous substance, paleblue with haematoxylin, stained deeply with alcian blue, but clearly could not be mucin because of its negative reaction with the Schiff reagent. It has been claimed that carboxymethyl cellulose gives a metachromatic reaction with tartaric acid-thionine, but the contents of the granuloma did not show metachromasia. The whole of the acellular part of the granuloma was dusted with haematoxylin-staining granules from disintegrated cells.

The innermost cellular layer was composed of macrophages with ill-defined, faintly eosinophilic cytoplasm. Some of these cells formed the giant cell clusters. A brown-yellow, non-iron-containing pigment was present in some macrophages of the outermost parts of the granuloma. The other cells of the inflammatory infiltrate consisted of a mixture of lymphocytes, plasma cells, and polymorphonuclears, with appreciable numbers of eosinophils. The inflammatory zone extended for a distance of nearly $1 \mathrm{~mm}$. beyond the limits of the non-cellular centre. Fibroblasts were evident in the outer parts of the granuloma and the formation of reticulin fibres was well advanced. The silver impregnations for reticulin, viewed in combination with elastic stains to define the original anatomy, conclusively demonstrated the association of the granulomas with centrilobular emphysematous spaces (Fig. 7). Reticulin fibres were abundant on all sides of the granulomas except those contiguous with the spaces. Here fibres were fewer and the cellular reaction was less intense, as would be expected if granulomas developed on the surface of spaces and became incorporated in the walls.

Necropsy Findings.-At the site of closure of the left upper lobe bronchus was a small fistula communicating with the left pleural cavity. The bronchus to the lower lobe contained haemorrhagic fluid. Within the left pleural space was a small quantity of blood-stained fluid and the remaining lobe was collapsed, solid, and purple. Section of this lobe, which was almost totally airless, revealed a diffuse red surface, with a few centrilobular emphysematous spaces as the only distinguishable feature. The pulmonary vein draining the lobe was normal, but the pulmonary artery was kinked and slightly narrowed by the tension of sutures which had been applied to the lung root.

The principal histological changes ranged from complete to partial infarction. The tissues had been invaded secondarily by cocci and Aspergillus. Mediumsized arteries and veins were often occluded by recent thrombus. The lobe had only partially expanded, but emphysematous spaces tended to be somewhat better expanded than the rest of the tissue. All but the largest centrilobular emphysematous spaces in the lobe were flooded by haemorrhagic oedema fluid obscuring the granuloma formation in haematoxylinand-eosin preparations. Granulomas became identifiable only after silver impregnation of the reticulin. 


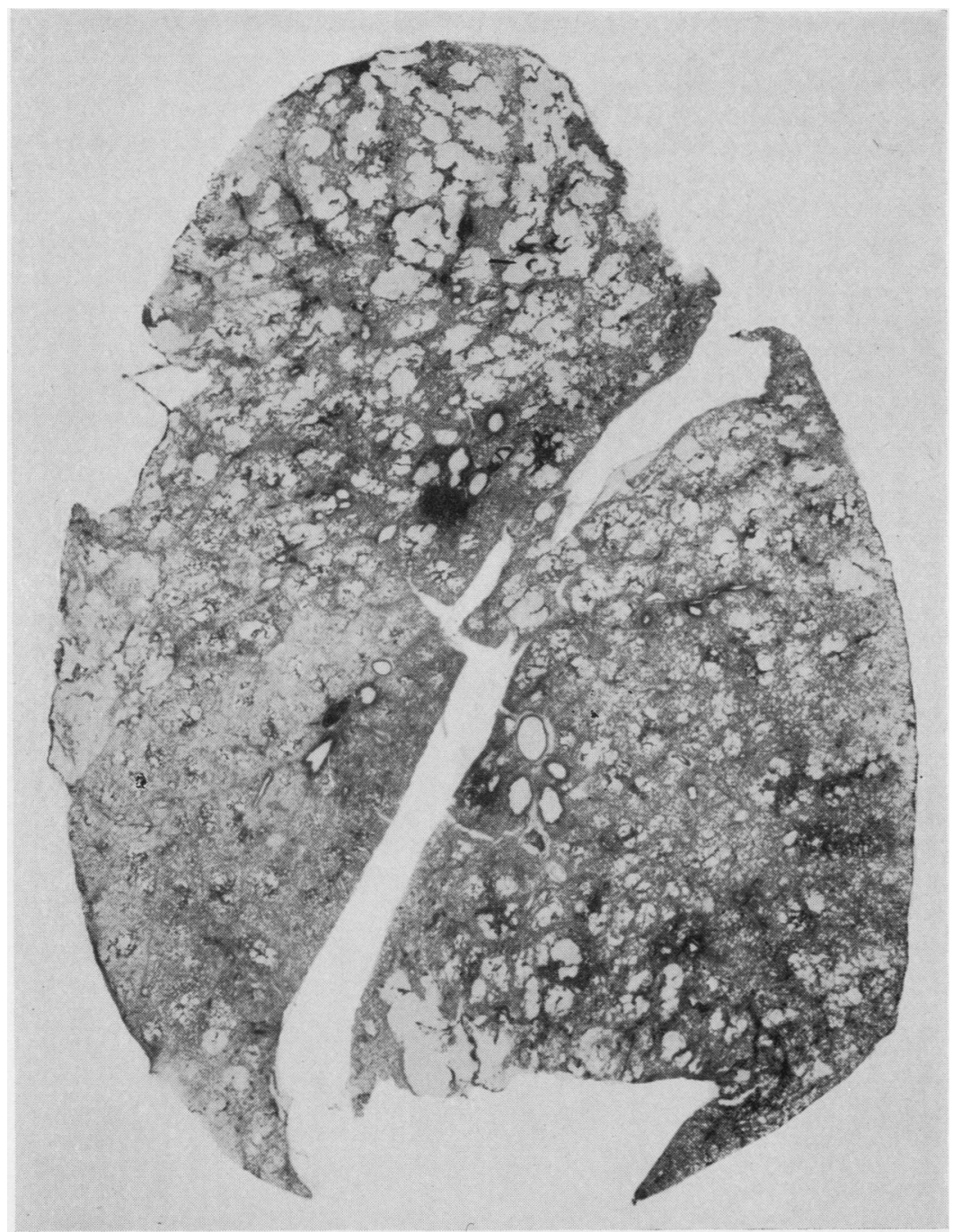

Fig. 3.-Large section (Gough and Wentworth) of right lung. Widespread centrilobular emphysema with spaces up to $1 \mathrm{~cm}$. diameter in upper lobe, $\times 2 / 3$.

They were few and differed from those in the resected lobe in showing no residue of crystalline material.

Right Lung.-This was moderately bulky, fully expanded, and emphysematous. To assist study of the emphysema it was fixed by infusion of formalin into the bronchus before cutting. Emphysematous spaces up to a size of nearly $1 \mathrm{~cm}$. were moderately numerous, widespread, and well demarcated from the bulk of the lung parenchyma (Fig. 3). In the latter, alveoli were generally thin-walled, only slightly larger than normal, and in normal anatomical arrangement (Fig. 8). The emphysematous spaces conformed to the pattern of centrilobular emphysema, as described by Leopold and Gough (1957). The identifying characteristic was the finding of emphysematous spaces, in which all normal arrangement of tissue had been destroyed, replacing the position in the normal anatomy where a branching system of respiratory bronchioles bridges the air pathway from the terminal bronchiole to the alveolar ducts (Fig. 8). This type of destruction of respiratory bronchioles seems to result from the spread of a bronchial inflammatory disease. In this case, fibrosis of the tissues in the centrilobular lesions was the evidence supporting the claim. Serial sections showed that the parent bronchioles of the lesions were of normal diameter. Pigmentation by dust was only slight throughout the lung, but it was concentrated in the tissue of the 


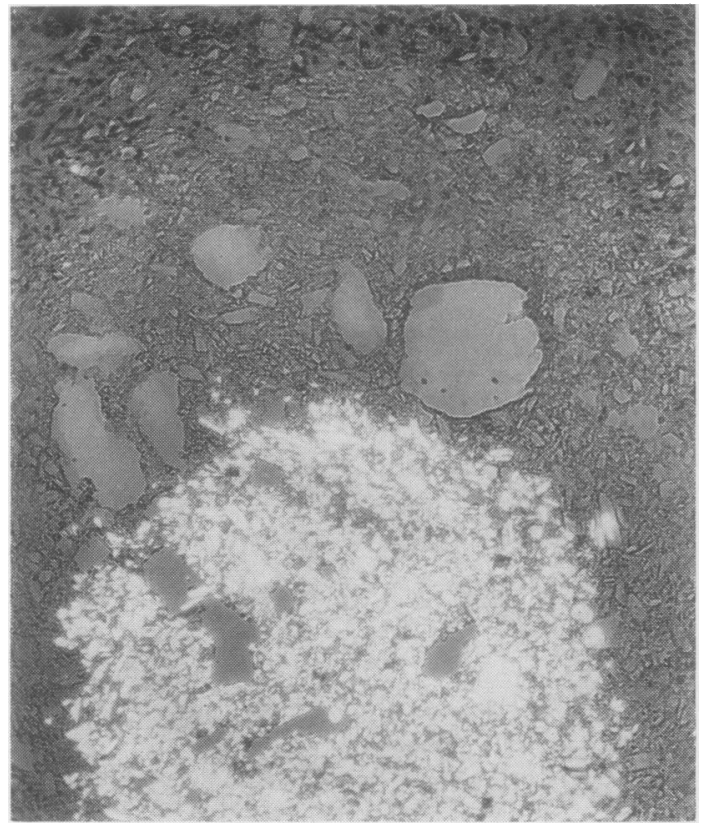

FIG. 4.-Crystalline content of granuloma with halo of acellular material, partly masses of a homogenous nature from resected lobe. Frozen section $\times 100$. Exposure with crossed Nicol prisms superimposed on ordinary light exposure.

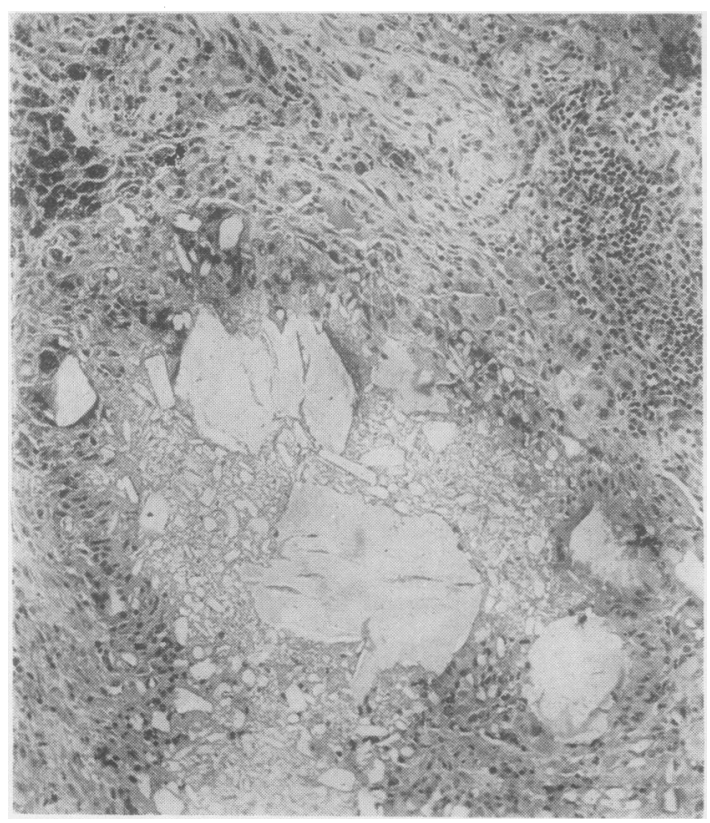

FIG. 6.-Resected lobe showing multinucleate giant-cells enclosing some of the pale blue-stained, homogenous material. Paraffin section $\times 100$. Haematoxylin and eosin.

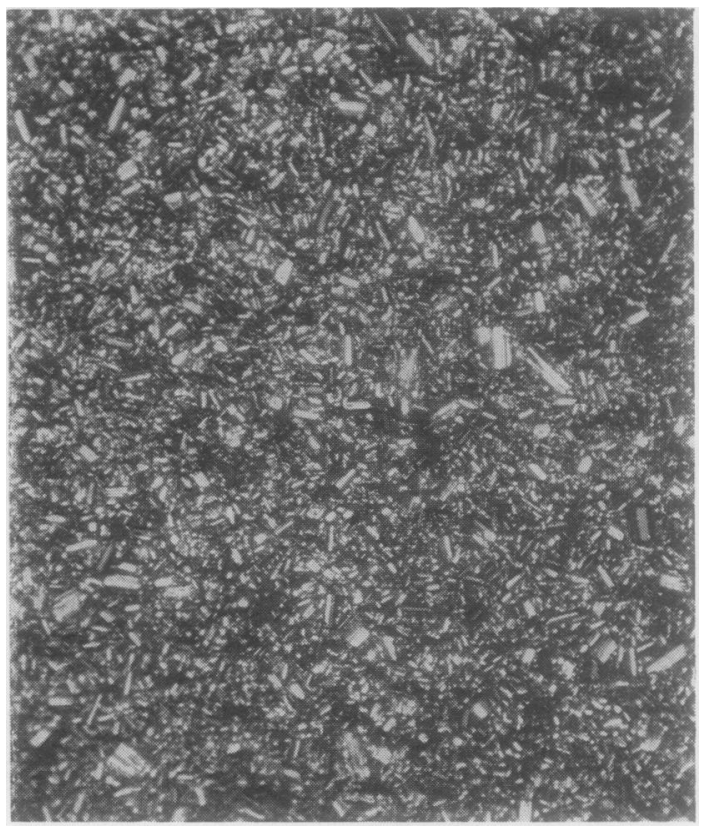

FIG. 5.-Wet preparation $\times 100$ crossed Nicol pr.sms. Quarter dilution of " dionosil aqueous."

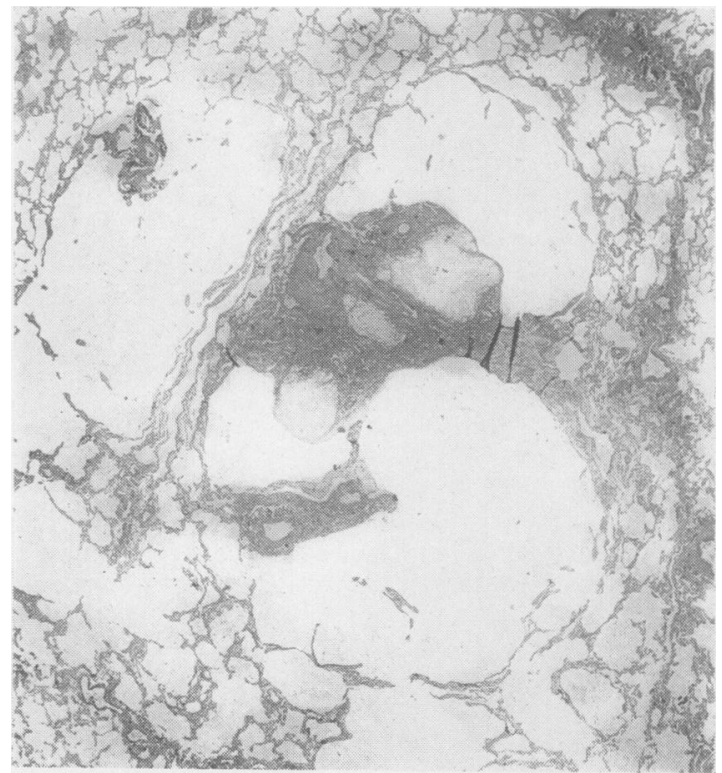

FIG. 7.-Moderately well-expanded area in the resected lobe. Three granulomas are seen, each with a central non-fibrous core. Two clearly show incorporation into the walls of contiguous centrilobular spaces. $\times 10$. Reticulin impregnation. 


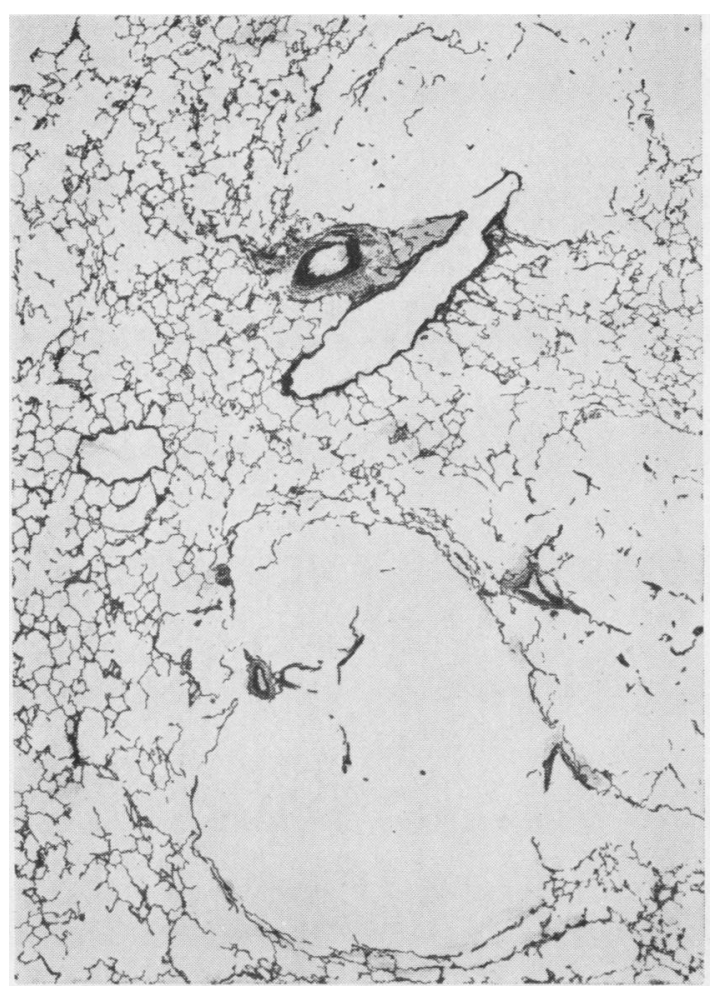

FIG. 8.-Two small centrilobular emphysematous spaces in the right lung. The communicating airways protrude into them. These unsupported airways probably collapse under the pressure of expiration. $\times 10$. Haematoxylin and eosin.

emphysematous areas, as would be expected, since centrilobular emphysema and dust deposition occur in relation to respiratory bronchioles.

Intense acute suppurative inflammation was apparent in the major bronchi but was only patchy in the bronchioles. In the former much of the superficial mucosa had been ulcerated. There was little evidence of a persisting chronic inflammation in the peripheral bronchial tree although mucosae were fibrous. The lining epithelium was cuboidal rather than columnar, and it contained very few goblet cells. The changes were principally those of fibrosis, atrophy, and dilatation, rather than active chronic inflammation.

No residual tumour was found in the body and the heart was normal.

\section{Discussion}

Two "dionosil" preparations are in common use. "Dionosil aqueous," which is a $50 \% \mathrm{w} / \mathrm{v}$ suspension of crystalline propyliodone ( $n$ propyl 3:5-di-iodo-4 pyridone-N-acetic acid) in sodium carboxymethyl cellulose (cellulose sodium glycollate), and "dionosil oily," in which the suspension is made in arachis oil. Both have an advantage over lipiodol in that the propyliodone is usually incleared from the lung in three to four days, even "should "alveolar filling" occur (Joynt and Harnick, 1955). Propyliodone is split by tissue esterases into an alcohol and the ion 3:5 di-iodo4-pyridone- $\mathrm{N}$-acetate which is excreted in the urine (Tomich, Basil, and Davis, 1953).

With the introduction of aqueous preparations, and particularly those containing propyliodone, it might have been hoped that the hazard of residual granulomas, present when oil-based media are used for bronchography (Felton, 1953), would be eliminated. However, it is important not to lose sight of the fact that, regardless of the choice of bronchographic medium, elimination of the compound primarily depends upon its expulsion in the bronchial secretions, and probably any part left in alveoli is accessible to removal only by tissue macrophages.

Vischer (1951) and Werthemann and Vischer (1951) reported granulomas following the use of a contrast medium with a water-miscible base in man, rats, and guinea-pigs. They attributed the reactions to the carboxymethyl cellulose in which the suspensions had been made, and not to the iodine compound, diodone. After examining 26 specimens removed six to 67 days after the use of the same bronchographic material, Zollinger (1951) disagreed with that finding. He contended that the material interpreted by Vischer as carboxymethyl cellulose, on the basis of its staining with tartaric acid-thionine, was mucin. He believed the lesions in question were mucin granulomas and insisted that carboxymethyl cellulose could not be distinguished from mucin on the tests used. Differentiation was, however, easily possible because of the negative staining of carboxymethyl cellulose with Schiff's reagent, and Zollinger with Fischer (1953) reported granulomas in which the presence of carboxymethyl cellulose had been proved to their satisfaction. They were found in three of 100 surgical specimens they examined.

Whether our instance of granulomas following the use of "dionosil aqueous" is due to the carboxymethyl cellulose or the propyliodone crystals is uncertain. It might be thought, on the basis of the staining reactions we have described, that the homogenous blue-staining material in the giant-cells is carboxymethyl cellulose. Hueper (1945), describing experimental thesaurosis with it, found a blue staining of the compound with haematoxylin, and Dunbar, Skinner, Wortzman, and Stuart (1959), in experiments with "dionosil 
aqueous" introduced intratracheally, also saw material with the same staining characteristics. The solubility of carboxymethyl cellulose in water varies with the stage to which the cellulose has been etherified, but experiment shows that the variety used in "dionosil aqueous" is readily soluble in cold water. Hence the fact that bluestaining deposits are seen in sections stained with no special precaution to exclude contact with water means either the deposits are not carboxymethyl cellulose or else its solubility has become changed. Vischer (1951), Werthemann and Vischer (1951), Zollinger and Fischer (1953), and Dunbar et al. (1959) did not exclude water from their preparations although they claim to identify carboxymethyl cellulose in the tissue sections.

The direct effect of the massive collections of crystalline, unesterified, propyliodone cannot be overlooked, and it would indeed be surprising if, after an interval of 14 to 15 days, such collections of particulate material did not evoke an inflammatory response. It may at this stage be reasonable to suppose that granulomas following the use of "dionosil aqueous" are only likely to result when conditions allow the retention in lung tissue of large collections of the medium, as occurred with "peripheral pooling" in the case of centrilobular emphysema described. In our view, the result is not to be taken as criticism of the material used. We believe that, in similar circumstances, the effect would probably be the same no matter how innocuous an agent could be evolved for use in bronchography.

The bronchograms demonstrate that there is free entrance into the centrilobular spaces from the bronchial tree, but there are many reasons why no appreciable evacuation or removal of the medium from the " pooled" areas occurred in the subsequent 14 to 15 days during which its course was followed. The anatomical arrangement in centrilobular emphysema (Fig. 8) consists of saccular spaces connected to the bronchioles by thin-walled passages entering at the margins and often protruding deeply into the spaces, and such an arrangement is unlikely to allow of free drainage and is ideal for causing check-valve closure of the passages on expiration. Removal of the aspirated contents must depend almost entirely on the activity of macrophages and enzymes. Felton (1953) observed that granulomas after the use of lipiodol were particularly common when the medium was retained in emphysematous regions, and, although he did not name it, some of the bronchograms he published suggest the appearance of "peripheral pooling." As a theoretical possibility it might be considered that emphysematous tissue would be less effective in its enzymatic esterification of propyliodone than healthy lung. The main point is, however, that only emphysematous lung and, in particular, the tissue of centrilobular spaces is liable to retain the bulky collections of contrast media that give the picture of "peripheral pooling." One might anticipate from the similar anatomical position of the emphysematous spaces in focal emphysema (Heppleston and Leopold) that it may also give "peripheral pooling." Even so, it is possible that granulomas would not result because, with the different type of connexion between the spaces and the bronchial tree, the medium may more freely drain away.

\section{SUMMARY}

Following bronchography with "dionosil aqueous" and the demonstration of "peripheral pooling," the "pooled" areas, which were best seen in the left upper lobe, remained visible until the resection of the lobe 15 days later for bronchogenic carcinoma. Pathological examination of the lobe showed granulomas containing the crystalline constituent of aqueous "dionosil" and a structureless material, possibly the carboxymethyl cellulose of the suspending medium. The granulomas were becoming incorporated in the walls of emphysematous spaces of the centrilobular variety. Necropsy examination of the opposite lung, which had not been outlined, showed it to be extensively diseased by centrilobular emphysema.

We thank Dr. H. M. Foreman and Mr. D. M. E. Thomas for permission to describe this case and Dr. J. G. Meek for the bronchograms. We are particularly grateful to Professor Gough, who encouraged our publishing the case and assisted in the preparation of the script. The photographs were taken by Mr. M. H. Travers.

\section{A P P E N D I X}

\section{Reactions for Carboxymethyl Cellulose}

The following tests were carried out on dried films of the suspending fluid of "dionosil aqueous" and deparaffinized sections of the granulomas. In the latter, attention was directed to the reaction of the homogenous material which stained a light slate colour with haematoxylin. Identical results were obtained with the dried films of cellulose from the "dionosil aqueous" and with the material contained in the granulomas.

1 Weak aqueous solutions of methylene blue resulted in a deep blue staining which, in the case of the material of the granulomas, was more intense than the staining of the surrounding cellular structures. 
2 Treatment with $0.2 \%$ anthrone (9,10-dihydro-9ketoanthracene) in concentrated sulphuric acid resulted, on warming, in a faint purple colour becoming yellow on dilution with water.

3 The carbazole test for carboxymethyl cellulose was negative.

\section{REFERENCES}

Dunbar, J. S., Skinner, G. B., Wortzman, G., and Stuart, J. R. (1959). Amer. J. Roentgenol., 82, 902.

Felton, W. L. (1953). J. thorac. Surg., 25, 530.
Heppleston, A. G., and Leopold, J. G. (1961). Amer. J. Med. In press. Hueper, W. C. (1945). Amer. J. Path., 21, 1021.

Joynt, G. H. C., and Harnick, L. R. (1955). Surg. Gynec. Obstet., $101,425$.

Leopold, J. G., and Gough, J. (1957). Thorax, 12, 219.

Simon, G., and Galbraith, H. J. B. (1953). Lancet, 2, 850.

Smith, W. G., Davies, F. R., Rothwell, P. G., Lodwig, G. S., and Mahoney, M. P. (1960). Ibid., 1, 515.

Tomich, E. G., Basil, B., and Davis, B. (1953). Brit. J. Pharmacol., 8, 166.

Vischer, W. (1951). Schweiz. med. Wschr., 81, 54.

Werthemann, A., and Vischer, W. (1951). Ibid., 81, 1077.

Zollinger, H. U. (1951). Ibid., 81, 210.

— and Fischer, F. K. (1953). Ibid., 83, 645. 